\title{
DVPW
}

\section{Förderpreise der Deutschen Vereinigung für Politische Wissenschaft}

Im Jahr 2007 schreibt die Deutsche Vereinigung für Politische Wissenschaft erneut zwei mit jeweils 1.000,00 Euro dotierte Förderpreise für politikwissenschaftliche Arbeiten aus. Ein Preis wird für eine Dissertation vergeben, ein weiterer für eine Monographie nach der Promotion.

Folgende Bedingungen gelten:

1. In Frage kommende Publikationen für beide Preise sollen 2006 veröffentlicht worden sein.

2. Dissertationen müssen mindestens mit magna cum laude bewertet sein.

3. Bewerberinnen und Bewerber dürfen zum Zeitpunkt der Bewerbung nicht zur Gruppe der Hochschullehrer und -lehrerinnen bzw. Gleichgestellter in fester Anstellung gehören. Bewerbungen von Juniorprofessorinnen und -professoren sowie von Kolleginnen und Kollegen, die eine Professur befristet vertreten, sind willkommen.

4. Der Kreis der Bewerber und Bewerberinnen ist auf Personen beschränkt, die in Deutschland promoviert wurden, oder Deutsche, die im Ausland promoviert wurden.

5. Für die beiden Förderpreise sind Selbstbewerbungen möglich; selbstverständlich sind Vorschläge von Dritten willkommen. Verlage sind nicht vorschlagsberechtigt.

6. Frauen sind besonders aufgefordert, sich zu bewerben; dies gilt auch für die Benennung von Kandidatinnen durch Dritte.

7. Die Einsendefrist für im Jahre 2006 veröffentlichte Publikationen ist der 15. Mai 2007.

Vorschläge und Benennungen für die Wissenschaftspreise bitten wir Sie, zusammen mit vier Exemplaren der Veröffentlichung und einer Kopie der Promotionsurkunde, an die Geschäftsstelle der DVPW zu senden. Vorstand und Beirat werden die Vorschläge durch eine aus drei Kolleginnen und Kollegen bestehende Jury begutachten lassen. Die Publikationen werden nach Abschluss des Verfahrens bei den Jurymitgliedern und evtl. in Anspruch genommenen Gutachter/innen verbleiben. Um Kosten zu sparen, wenden Sie sich daher am besten an den publizierenden Verlag und bitten diesen, uns vier Freiexemplare zu übersenden.

Deutsche Vereinigung für Politische Wissenschaft, c/o Universität Osnabrück, FB 1 Sozialwissenschaften, D-49069 Osnabrück, www.dvpw.de 